with a reduction in BPD (OR 0.797; 95\% CI 0.666-0.954; $\mathrm{p}=$ 0.013), severe HIV (OR 0.844; 95\% CI 0.734-0.970; $\mathrm{p}=$ 0.017 ), and an increase in survival (OR 1.384; 95\% CI 1.213$1.580 ; \mathrm{p}<0.001)$.

\section{P0-0681 EFFECTS OF EDUCATION NURSE PROGRAM IN IMPROVING FEEDING PATTERN IN PREMATURE INFANTS}

${ }^{1} \mathrm{~A}$ Beissel, ${ }^{1} \mathrm{KA}$ Nguyen, ${ }^{1} \mathrm{~F}$ Pillet, ${ }^{1} \mathrm{~F}$ Doiret, ${ }^{1} \mathrm{~F}$ Plaisant, ${ }^{1} \mathrm{H}$ Gauthier-Moulinier, ${ }^{1} \mathrm{AS}$ Magrou, ${ }^{1} \mathrm{H}$ Tarifa-Buisson, ${ }^{1} \mathrm{~F}$ Deruy, ${ }^{1} \mathrm{AS}$ Goyet, ${ }^{1} \mathrm{I}$ Conti, ${ }^{1} \mathrm{C}$ Keita, ${ }^{1} \mathrm{C}$ Duhamel, ${ }^{2} \mathrm{~S}$ Hommey, ${ }^{2} \mathrm{SDA}$ Hommey, ${ }^{2} \mathrm{~S}$ Touzet, ${ }^{1} \mathrm{O}$ Claris. ' 69 , Hôpital Femme Mère Enfant, Lyon, France; ${ }^{2} 69$, Pole Information Medicale Evaluation Recherche, Lyon, France

\subsection{6/archdischild-2014-307384.1321}

Background and aim Poor oral motor development in premature infants is common. These feeding problems lead to longer hospital stay and higher hospital cost. Different interventions have shown their efficacy to accelerate transition and move faster to full oral feeding autonomy. Our study aimed to evaluate the impact of a two year education nurse program on feeding pattern and hospital stay of premature neonates.

Method We conducted a prospective, interventional study during 3-years (2013-2015) in an NICU of a University hospital in Lyon, France. After a pre-interventional six-months period, nurses received a two-days theoretical session coupled to three practical workshops. This training focused on early oral feeding, oral motor re-education and non-nutritive suction. During this two-years study period, nurses are coached in routine practice by speech therapist, physiotherapist, and psychologist. A satisfaction inquiry is conducted at the end of the theoretical session. Finally, we will evaluate the impact on the age of full oral feeding autonomy and hospital stay in a 6 month post-intervention period compared to a pre-intervention period.

Primary results Three day sessions have been organised in 2014 as well as nine workshops with 55 health professionals participating ( $46 \%$ of the total nurse health staff). Hundred percent of the nurses were satisfied and thought that this formation was helpful and could change their practice.

Benefits This would be the first study in France evaluating an educational intervention to improve oral feeding problems in premature neonates.

\section{PO-0682 WITHDRAWN}

\section{P0-0683 COMPARISON OF CLINICAL CONDITIONS OF VLBW (VERY LOW BIRTH WEIGTH INFANTS) AND BODY TEMPERATURE AT ADMISSION IN THE NICU}

WAG Ferri, FE Martinez. Pediatria, Faculdade de Medicina de Ribeirao Preto, Ribeirao Preto, Brazil

\subsection{6/archdischild-2014-307384.1322}

Abstract In Neonatal Intensive Care Units (NICU) the estimative of risk of morbidity and mortality in preterm infants is important for clinical decisions and management. The temperature at ward admission is known as an important factor to predict outcome. VLBW infants have lower temperatures at NICU admission many times due to their need of resuscitation measures at birth. The lower their temperature at adimission the higher the

\begin{tabular}{llll} 
Abstract PO-0683 Table 1 & & \\
\hline & Temperature $\geq \mathbf{9 5 ^ { \circ }} \mathbf{F}$ & Temperture $<\mathbf{9 5 ^ { \circ }} \mathbf{F}$ & $\mathbf{p}$ \\
APGAR $\leq 5$ & $7,2 \%$ & $20 \%$ & 0,0001 \\
Resuscitation & $81,7 \%$ & $89,5 \%$ & 0,37 \\
Cardiac massage & $1,9 \%$ & $8,69 \%$ & 0,019 \\
Epinephrine & $1,1 \%$ & $3,4 \%$ & 0,0001 \\
Gestacional age & $29,5 \%$ & $27,6 \%$ & 0,0001 \\
SNAP-PE 2 & $23,2 \%$ & $50,5 \%$ & $<0,001$ \\
N & 263 & 115 & \\
\hline
\end{tabular}

SNAP-PE II score, that has a good predictive value in NICUs. Many clinical decisions, as early enteral feeding, assess these scores, often alone.

Objectives Evaluate the accuracy of the body temperature as an outcome predictor for VLBW infants in NICU.

Methods A retrospective study was carried out, using database. We evaluated 378 patients, VLBW infants born in our service, during the period January 2008 to December 2012. The exclusion criteria was major malformations. For analysis were performed "chi-square test" and Student's t-test.

Results and conclusions The results, after we analysed 378 patients, demonstrated the lower the temperature at the admission at NICU the worst outcome. The group with lower temperatures had lower birth weight and gestacional age and need of intensive neonatal ressuscitation at birth.

\section{PO-0684 EFFECTS OF ENDOTRACHEAL SUCTIONING IN NEWBORN PRETERM INFANTS WEIGHING BETWEEN 500 AND $1500 \mathrm{G}$}

ACE Pirone, W Gonçalves-Ferri, FE Martinez. Pediatrics, University of São Paulo, Ribeirão Preto, Brazil

\subsection{6/archdischild-2014-307384.1323}

Objective To observe different ways of implementing the techniques of aspiration of preterm infants and verify factors associated with changes in oxygen saturation and contamination of equipment.

Methods An observational, prospective, descriptive study. The aspirations of the endotracheal tube in infants with birth weights between 500 and $1500 \mathrm{~g}$ were observed during two procedures; morning and evening.

Results We evaluated 32 infants with a mean weight of $942 \mathrm{~g}$ and a mean gestational age of 28 weeks (64 aspiration procedures). During aspiration $61 \%$ of children had hypoxemia. The saturation drops were more related to the aspiration held by a single professional, also when they were made more than three disconnections of the fan and when disconnections occurred beyond fifteen seconds. The pre - oxygenation did not decrease significantly during the procedure hypoxia and preterm - oxygen showed hyperoxia from third minute of recovery. The aspiration held by two professionals joined the adequate pre - oxygenation, decreased number and duration of disconnections ventilation, and less contamination of materials. The use of saline during suctioning in less than $1 \mathrm{ml}$ volume was associated with significant decreases saturation.

Conclusions The aspiration of the endotracheal tube can lead to hypoxemia. To minimise episodes is important that it be performed by two professionals that meet the care disconnecting the system less than 3 times and use less than 15 seconds. Also pre-oxygenation should be done with caution and the use of less 
than $1 \mathrm{ml}$ volume of saline solution leads to negative consequences for the patient.

\section{PO-0685 OUTCOME OF THYROID FUNCTION SCREENING IN BABIES BORN TO MOTHERS WITH AUTOIMMUNE HYPOTHYROID DISEASE}

A Gupta, S Balogan, S Sarangpani, C Harikumar. Paediatrics, University Hospital of North Tees, Stockton-on-Tees, UK

10.1136/archdischild-2014-307384.1324

Background and aims Autoimmune problems in mums are known to affect the newborn due to transplacental passage of antibodies. It Is reported that maternal autoimmune hypothyroidism can lead to transient but potentially serious effects in newborn babies warranting treatment. This has led to the practice of screening babies to help in early diagnosis and treatment. Unfortunately clinical practice in this area still suffers from conflicting evidence.

We carried out this retrospective review of our practice of assessing neonatal thyroid function in maternal autoimmune hyperthyroidism to help inform our practice locally whilst contributing to the discussion towards building a consensus nationally and internationally.

Methods Newborn babies born between January 2012 to March 2014 to mothers with autoimmune hypothyroid problems had their thyroid functions checked on day 3 and 10 respectively. They were also monitored clinically for signs and symptoms of hypo/ hyperthyroidism.

Results Overall 31 babies were screened in the study period, of which none warranted treatment for transient or permanent hypo/hyperthyroidism. The review also highlighted difficulties in implementing this guidance, as majority of the babies did not get investigated as per schedule.

Conclusion In our experience, babies born to mothers with auto-immune hypothyroidism did not develop transient hypothyroidism. There is a need for a larger scale study to look at the possible adverse effects of maternal autoimmune thyroid problems in the newborn.

\section{P0-0686 THE IMPACT OF VISCERAL OSTEOPATHIC TREATMENT ON THE MECONIUM EVACUATION IN VERY LOW BIRTH WEIGHT INFANTS}

N Haiden, A Kreissl, B Pimpel, A Berger. Pediatrics, Medical University of Vienna, Vienna, Austria

\subsection{6/archdischild-2014-307384.1325}

Objective To determine whether the complementary approach of manipulative osteopathic treatment accelerates complete meconium excretion and improves feeding tolerance in very low birth weight infants.

Methods This study was a prospective, randomised, controlled trial in premature infants with a birth weight $1500 \mathrm{~g}$ and a gestational age 32 weeks who received a visceral osteopathic treatment algorithm 3 times during their first week of life or no treatment.

Results Passage of last meconium occurred after a median of 7.5 days $(95 \%$ confidence interval: $6-9$ days, $\mathrm{n}=20$ ) in the intervention group and after 6 days (95\% confidence interval: 5-9 days, $\mathrm{n}=21)$ in the control group $(\mathrm{p}=0.11)$. However, osteopathic treatment was associated with a 12 day longer time to full enteral feedings $(\mathrm{p}=0.02)$, and a longer hospital stay (44 days longer in the intervention group; n.s). Osteopathic treatment was tolerated well and no adverse events were observed.

Conclusions Visceral osteopathic treatment oft the abdomen did not accelerate meconium excretion in VLBW-infants. However infants in the osteopathic group had a longer time to full enteral feedings and a longer hospital stay what must be interpreted as negative side effect. Further investigations are needed with modified protocols focussed on cranial osteopathy in this vulnerable group of patients. Currently the application of visceral osteopathic techniques cannot be recommended in VLBW-infants without further clinical trials.

\section{PO-0687 ALCOHOL USE IN PREGNANCY: GUIDELINES ON PRENATAL ARE SUFFICIENT?}

A Harff, S Nader, P Nader, S Fiamenghi. Neonatology, ULBRA/SSMD University Hospital, Canoas, Brazil

\subsection{6/archdischild-2014-307384.1326}

Background Alcohol use during pregnancy and the effects of exposure to the fetus have been described. The effects occur independent of the quantity consumed. Objective is identify alcohol use during pregnancy and check the orientation being given about the risk of alcohol use during prenatal care.

Design/methods 302 postpartum women were interviewed in the University Hospital ULBRA/Mãe de Deus from March to August 2012. We used the validated questionnaire T - ACE (Tolerance, Annoyed, Cut down, and Eye- opener) on the postnatal room pairs, featuring a random sample. The women were also asked about the performance of pre-natal and present guidelines on the use of alcohol.

Results Alcohol use during pregnancy was reported by 24\%. When used T - ACE was positive in $33.1 \%$ of subjects. During prenatal care, 219 women were instructed in prenatal and of these 72 (33\%) had positive T - ACE. Since the 68 women who were not oriented, 26 (35\%) are positive T-ACE group. No significance was found to have been targeted during the prenatal or not. Significant difference between the means of alcohol abuse $(\mathrm{T}-\mathrm{ACE})$ and the reported use during pregnancy $(\mathrm{p}<0.001)$, being higher in the instrument.

Conclusion It can be concluded that alcohol abuse found through validated instrument is high among pregnant women at the ULBRA/SSMD University Hospital. There was no difference in alcohol abuse among mothers counselled on the risk of alcohol use during pregnancy and not targeted during the prenatal period.

\section{PO-0688 ESTABLISHING PARENTHOOD IN THE BORDERLAND OF NORMALCY AFTER MODERATE OR LATE PRETERM BIRTH}

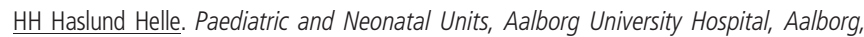
Denmark

\subsection{6/archdischild-2014-307384.1327}

Aim To investigate the process of establishing parenthood after birth of moderate or late preterm infant (GW 32-37).

Background Moderate or late preterm counts $80 \%$ of the premature population. In spite of known morbidity they are discharged without specialised health care services. Parents can 